\title{
ESTUDOS DE RELAÇÕES ESTRUTURA-ATIVIDADE QUANTITATIVAS (QSAR) DE BIS-BENZAMIDINAS COM ATIVIDADE ANTIFÚNGICA
}

\author{
Vera L. de Almeida, Júlio César Dias Lopes, Sheila Rodrigues Oliveira e Claudio L. Donnici* \\ Departamento de Química, Instituto de Ciências Exatas, Universidade Federal de Minas Gerais, Av. Antônio Carlos, 6627, 31270 - \\ 901 Belo Horizonte - MG, Brasil \\ Carlos A. Montanari \\ Departamento de Química e Física Molecular, Instituto de Química de São Carlos, Universidade de São Paulo, Av. Trabalhador \\ São-carlense, 400, 13560-970 São Carlos - SP, Brasil
}

Recebido em 5/10/09; aceito em 26/3/10; publicado na web em 20/7/10

\begin{abstract}
QUANTITATIVE STRUCTURE-ACTIVITY RELATIONSHIPS (QSAR) STUDIES OF BISBENZAMIDINES WITH ANTIFUNGAL ACTIVITY. This paper describes 2D-QSAR and 3D-QSAR studies against Candida albicans and Cryptococcus neofarmans for a set of 20 bisbenzamidines. In the studies of 2D-QSAR with C. albicans it was obtained a correlation between $\log \mathrm{MIC}^{-1}$ and lipolo component-Z $\left(\mathrm{r}^{2}=0.68 ; \mathrm{Q}^{2}=0.51\right)$. In the case of C. neofarmans a correlation between $\log \mathrm{MIC}^{-1}$ and lipolo component-Z and of Balaban index $\left(r^{2}=0.85 ; Q^{2}=0.6\right)$ was obtained. 3D-QSAR studies using CoMFA showed that the steric fields contributed more to the predicted activities for Candida albicans (94.9\%) and Cryptococcus neofarmans (97.9\%).
\end{abstract}

Keywords: QSAR; bis-benzamidines; antifungal activity.

\section{INTRODUÇÃO}

As infecções causadas por fungos são, geralmente, limitadas à superfície dos tecidos e são relativamente fáceis de serem curadas. ${ }^{1,2}$ Com os avanços da medicina, o número de pacientes em internação hospitalar prolongada cresceu muito, assim como a população de pacientes imunologicamente vulneráveis a fungos e bactérias, levando a um aumento da frequência de infecções causadas por patógenos oportunistas. Tais internações propiciam uma maior exposição do paciente a patógenos multirresistentes, comumente presente nos centros hospitalares. ${ }^{3,4}$ Adicionalmente, em pacientes imunossuprimidos as infecções causadas por fungos podem ser graves. ${ }^{5,6}$ Ao longo dos últimos 10 anos, a incidência de infecções causadas por fungos tem aumentado muito em número e em gravidade. Para piorar este grave quadro epidemiológico, o uso incorreto de antibióticos de amplo espectro de ação tem levado ao surgimento de populações de microorganismos resistentes a estes agentes quimioterápicos, ${ }^{7}$ apesar da maior parte dos fungos patogênicos serem muito bem conhecidos tanto quanto o seu tratamento clínico..$^{3-9} \mathrm{O}$ tratamento destas infecções fúngicas invasivas (IFIs) e oportunistas permanece ainda insatisfatório com o uso da maior parte dos agentes antifúngicos usuais, tais como itraconazol e anfotericina $\mathrm{B}$, e além disso os agentes patogênicos oportunistas têm aumentado cada vez mais a sua resistência mesmo com o uso de novos agentes antifúngicos como, por exemplo, o voriconazol. Um estudo relatou que em 47 a $68 \%$ dos pacientes não houve eficácia terapêutica. ${ }^{89}$ Diante deste grave e crescente problema de saúde, o governo americano estabeleceu uma comissão nacional para o estudo e a prevenção das doenças microbianas resistentes Antimicrobial Resistance Prevention Initiative (ARPI) - cuja principal meta é auxiliar no desenvolvimento de novos agentes antimicrobianos mais eficientes. ${ }^{10-13}$

O gênero Candida é responsável por cerca de $80 \%$ das infecções fúngicas em ambiente hospitalar e constitui causa relevante de infecções de corrente sanguínea. ${ }^{14}$ Entre as espécies mais invasivas, que são responsáveis por quadros graves de candidíases, incluem-se $C$.

*e-mail: cdonnici@terra.com.br glabrata, C. parapsilosis e C. krusei ${ }^{15}$ Cryptococcus neofarmans é o segundo mais comum fungo oportunista em pacientes HIV positivos. A manifestação clínica desta infecção irá depender do sistema imunológico do hospedeiro. ${ }^{16}$

Entre os agentes antifúngicos descritos na literatura pode-se destacar a pentamidina, que é o fármaco de escolha no tratamento da PCP (pneumonia causada por Pneumocistis carinii) e que tem sido usada clinicamente no tratamento de tripanossomíases e leishmaniose. ${ }^{17-20}$ A pentamidina e os seus análogos também se mostraram ativos contra Plasmodium falciparum, Cryptosporidium parvum, Toxoplasma gondii, Giardia lambia, Leishmania mexicana, Leishmania amazonensis, Aspergillus fumicatus, Fusarium soloni e várias espécies de Candida. ${ }^{21-23}$

Várias hipóteses têm sido propostas para explicar o mecanismo de ação farmacológica das amidinas aromáticas. Segundo estudos biofísicos, que incluem RMN e cristalografia de raios X, estes compostos se ligam na fenda menor do DNA em regiões ricas em adenina-timina (A-T) no código genético. ${ }^{24}$ Eles possuem características estruturais em comum que incluem: podem assumir uma conformação isoélica ao DNA, possuem grupos amidínicos carregados positivamente em $\mathrm{pH}$ fisiológico. ${ }^{17} \mathrm{~A}$ estrutura complementar à dupla hélice do DNA aumenta a superfície de contato e alinha grupos que favorecem a interação com o DNA. ${ }^{25}$ Estudos prévios de modelagem molecular e relação estrutura-atividade utilizando compostos amidínicos têm mostrado a importância da estrutura isoélica e da carga positiva presente nestes compostos para a interação com a fenda menor do B-DNA. ${ }^{18,26-28}$ Estudos de modelagem molecular sugerem ainda que o grupo amidínico desses compostos forme ligações de hidrogênio com os átomos receptores das bases A-T presentes na fenda menor. ${ }^{17,22,26}$ Há fortes evidências de que eles levariam à subsequente inibição de enzimas DNA-dependentes. ${ }^{23,29,30}$

Assim, a pesquisa e desenvolvimento de novos agentes antimicrobianos e/ou a modificação molecular de compostos químicos já existentes para descoberta de novos compostos-protótipo com atividade biológica potencial são importantes ferramentas para se garantir o combate ao crescente número de fungos patogênicos resistentes e levar ao uso clínico de compostos cada vez mais eficientes e menos 
tóxicos. O uso de quimioinformática e técnicas de QSAR-2D e 3D tem contribuído para o planejamento de novos potenciais agentes antifúngicos. ${ }^{31}$

As análises de QSAR têm como principal objetivo a construção de modelos matemáticos que relacionem a estrutura química à atividade biológica de uma série de compostos análogos. Em geral, esses compostos diferem entre si pela presença de um ou mais grupos substituintes em posições definidas na estrutura química comum à série. ${ }^{32,33}$ Esses modelos devem ser capazes de explicar o fenômeno observado bem como de proporcionar previsões dentro e, se possível, fora dos limites observados, ${ }^{34}$ sendo utilizadas técnicas estatísticas multivariadas que podem gerar modelos lineares ou não. Para os modelos lineares, as técnicas de Regressão Múltipla Linear (RML ou Multiple Linear Regression - MLR) e Mínimos Quadrados Parciais (MQP ou PLS - Partial Least Squares) são as funções matemáticas mais utilizadas. ${ }^{35}$

Neste trabalho foram utilizadas análises de QSAR-2D e 3D. Esta última foi realizada através da análise comparativa do campo molecular (Comparative Molecular Field Analysis- CoMFA) de um banco de compostos bisbenzamidínicos obtidos da literatura, ${ }^{36,37}$ utilizando o software SYBYL 6.7. ${ }^{38}$ No método CoMFA ${ }^{38-40}$ as energias de interação eletrostática e estereoquímica entre uma sonda e a molécula ${ }^{18,40,41}$ foram usadas como descritores. Para o cálculo de interação eletrostática foi utilizado o potencial coulômbico e para o cálculo dos campos estereoquímicos foi utilizado o potencial de Lennard-Jones. Foram gerados mapas de contorno que permitem uma análise visual das propriedades moleculares relevantes para a atividade biológica buscada. ${ }^{18}$

\section{PARTE EXPERIMENTAL}

\section{Geração da estrutura 3D}

Inicialmente foi construído um banco de dados com 19 compostos benzamidínicos substituídos (Figura 1) obtidos da literatura com valores quantitativos de atividade antifúngica (concentração inibitória mínima) frente à Candida albicans e Cryptococcus neofarmans relatados na literatura. ${ }^{36,37}$ Todos esses compostos se ligam à fenda menor do B-DNA em regiões ricas em adenina-timina (A-T). ${ }^{21,23,41-45}$

As moléculas foram construídas na sua forma protonada utilizando o programa SYBYL 6.7. As cargas parciais dos átomos dos compostos foram calculadas utilizando o programa MOPAC 7.0 por métodos semiempíricos de cálculo Hamiltoniano MNDO-ESP. ${ }^{26,46,47}$

Utilizando o programa MacroModel versão $5.5^{48}$ os complexos DNA-ligante foram construídos utilizando o octâmero do B-DNA

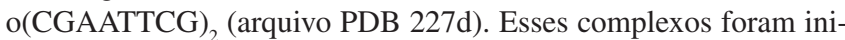
cialmente minimizados, mantendo o DNA rígido e o ligante flexível. Foram feitos 2500 ciclos utilizando o campo de força Amber, ${ }^{49}$ tratamento eletrostático dependente da distância e constante dielétrica molecular simulando a presença de água. A minimização foi feita utilizando gradiente conjugado. As distâncias de corte para o cálculo das interações foram padronizadas em $20 \AA$ para interação coulômbica e $8 \AA$ em van der Waals (critérios de convergência: $0,01 \mathrm{kcal} /$-mol). Os complexos DNA-ligante obtidos foram então otimizados usando o procedimento MM-DM-MM (mecânica molecular-dinâmica molecular-mecânica molecular) no programa MacroModel 5.5, considerando o DNA e os ligantes flexíveis, utilizando o campo de força Amber. ${ }^{21,23,39-43}$ As condições de minimização utilizando mecânica molecular descritas acima foram seguidas. O complexo de menor energia obtido foi submetido à dinâmica molecular com o DNA e o ligante flexíveis. A dinâmica foi feita em três etapas, com temperatura constante de $300 \mathrm{~K}$. Na primeira etapa, o tempo total de dinâmica foi de 100 picossegundos com passos de 1,5 femtossegundos. Na segunda
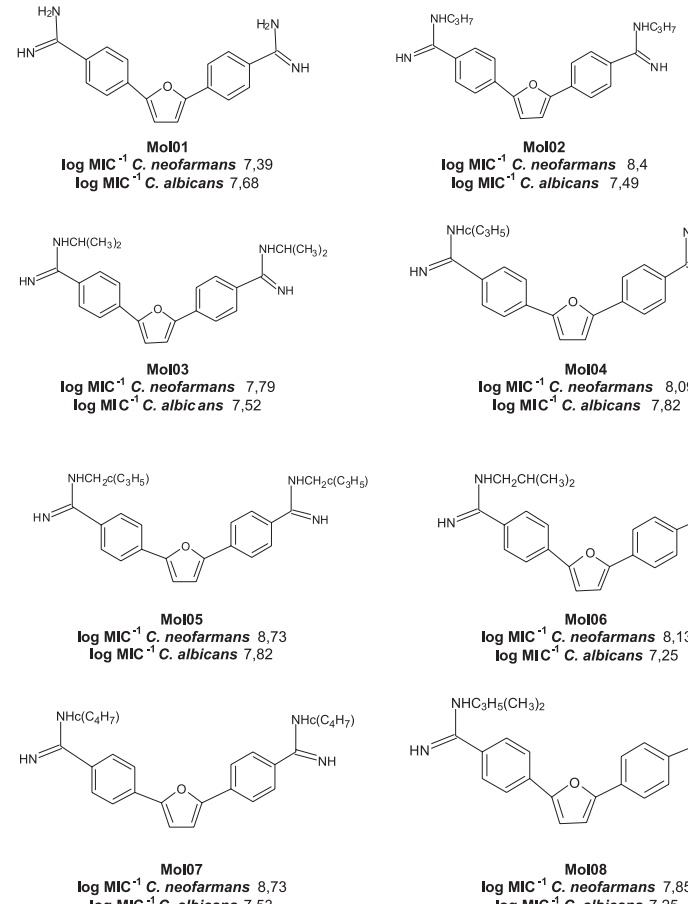

$\log \mathrm{MIC}^{-1}$ C. neofarmans 8,4
$\log \mathrm{MIC}^{-1}$ C. albicans 7,49
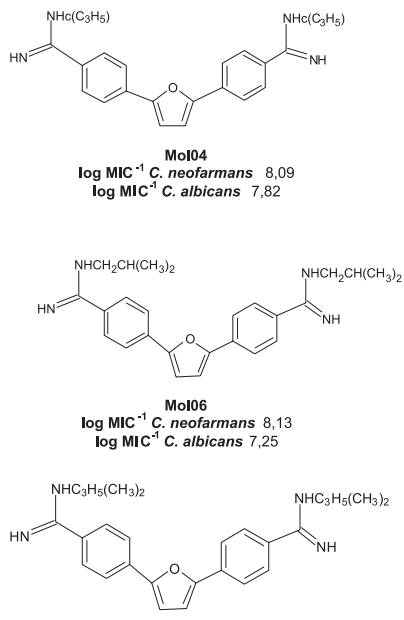

$\log$ MIC $^{-1} C$. albicans 7,53
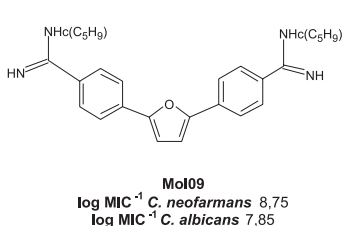

$\log \mathrm{MIC}^{-1}$ C. neofarmans 8,75
$\log \mathrm{MIC}^{-1}$ C. albicans 7,85

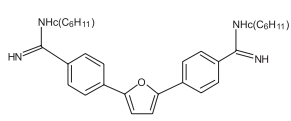

Mol11
$\log$ MIC $^{-1}$ C. neofarmans 8,48
$\log$ MIC $^{-1}$ C. albicans 7,57

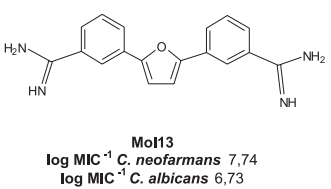

Mol08
$\log$ MIC $^{-1}$ C. neofarmans 7,85 $\log M I C^{-1}$ C. neofarmans 7,85
$\log$ MIC ${ }^{-1} C$. albicans 7.25
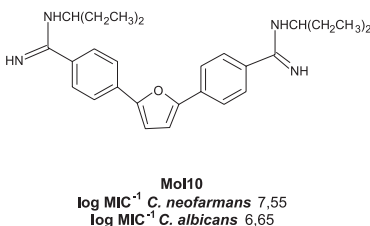

$\log \mathrm{MIC}^{-1}$ C. neofarmans 7,5

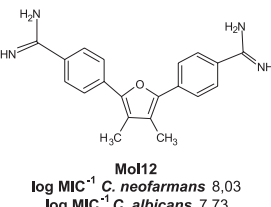

$\log \mathrm{MIC}^{-1}$ C. neofarmans 8,03
$\log \mathrm{MIC}^{-1}$ C. albicans 7,73
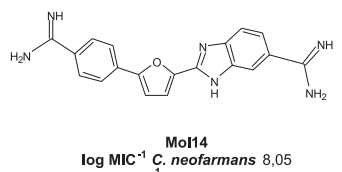

$\log \mathrm{MIC}^{-1}$ C. neofarmans 8,05

$\log$ MIC $^{-1} C$. albicans 6.73
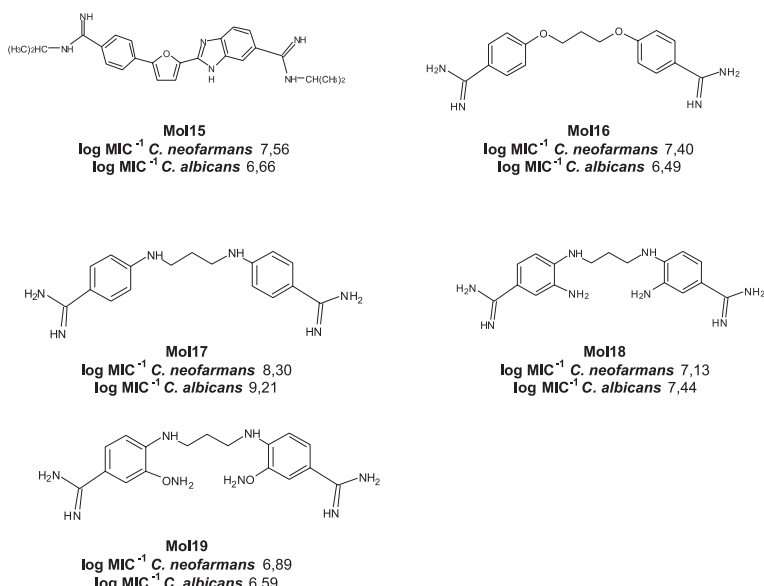

Figura 1. Estruturas das bisbenzamidinas do banco de dados e a atividade biológica de frente aos fungos, Cryptococcus neofarmans e Candida albicans ${ }^{42,43}$ 
etapa o tempo total de dinâmica foi de 300 picossegundos com passos de 1,5 femtossegundos. Na terceira etapa o tempo total de dinâmica foi de 500 picossegundos com passos de 1,5 femtossegundos. O complexo de menor energia foi submetido novamente à mecânica molecular utilizando as mesmas condições descritas anteriormente. Após a minimização, os complexos foram desfeitos.

As conformações de algumas bisbenzamidinas obtidas na docagem manual foram avaliadas por comparação com aquelas conformações obtidas por cristalografia de raios $\mathrm{X}$ depositadas no Protein Data Bank- PDB. ${ }^{50-52}$ As moléculas 3 (arquivo PDB 298D), 4 (arquivo PDB 289D), 7 (arquivo PDB 1EEL) e 11 (arquivo PDB 1FMS) foram comparadas através do cálculo de desvio médio quadrático (RMS Root Mean Square) utilizando o programa INSIGTH II versão $97 .{ }^{53}$

\section{Estudos de QSAR-2D frente ao fungo Candida albicans}

Utilizando o programa Tsar versão $3.21,{ }^{54}$ as moléculas na conformação obtida do docking manual foram alinhadas utilizando alinhamento baseado na massa e na carga atômica (massa peso $=1$, carga peso $=10$ ). Cinquenta parâmetros físico-químicos (estereoquímicos, eletrônicos, topológicos, lipofílicos e de similaridade) foram calculados e normalizados segundo procedimento média/desvio. Foi realizada análise de RML usando o programa BILIN, ${ }^{55}$ utilizando como grupo de treino os compostos $1,2,3,4,5,10,11,12$ e 13 (Figura 1) e como grupo de teste os compostos 6, 7, 8 e 9 (Figura 1) que foram escolhidos de forma aleatória. Como variável dependente foi utilizado $\log \mathrm{MIC}^{-1}$ frente ao fungo Candida albicans e como variáveis independentes aqueles calculados usando programa Tsar versão 3.2. ${ }^{53} \mathrm{O}$ método de validação cruzada escolhido foi Leaving One Out (LOO). A equação que correlaciona atividade biológica com parâmetros físico-químicos foi obtida e feita uma predição dos valores de $\log \mathrm{MIC}^{-1}$ dos compostos do grupo de treino. A atividade biológica dos compostos do grupo de teste e das moléculas da validação lateral, assim como as porcentagens de erro desta análise, foram calculadas para cada composto.

\section{Estudos de QSAR-2D frente ao fungo Cryptococcus neofarmans}

Utilizando-se a mesma metodologia descrita anteriormente, foi feita uma regressão múltipla linear, programa BILIN, ${ }^{54}$ utilizando como grupo de treino os compostos $3,4,5,7,8,10,11,12$ e 13 (Figura 1) e como grupo de teste os compostos 1, 2, 6, e 9 (Figura 1) escolhidos de forma aleatória.

\section{Estudos de QSAR-3D frente aos fungos Candida albicans e Cryptococcus neofarmans}

Na análise de QSAR-3D foi utilizado o programa CoMFA, do pacote de programas SYBYL 6.7. ${ }^{36}$ As moléculas do banco de dados (Figura 1) foram alinhadas pelo método ponto a ponto com estrutura da pentamidina obtida por cristalografia raios X $(227 \mathrm{~d}$ arquivo PDB). Os campos eletrostático e estereoquímico do CoMFA foram calculados em cada ponto de interseção da caixa usando as funções potencial de Leonard Jones e Coulômbica do campo de força Tripos utilizando diferentes sondas. Com base no conhecimento da interação destes compostos com a fenda menor do BDNA, foram escolhidas sondas que fossem capazes de mimetizar a interação da droga com o alvo biológico: a sonda 02 (oxigênio sp2) aceptor de ligação de hidrogênio, sonda 03 (oxigênio sp3) aceptor de ligação de hidrogênio, Nar (nitrogênio sp2 pertencente a anel aromático), $\mathrm{CH}^{+1}$ (sonda default do programa), N3 (nitrogênio sp3) aceptor de ligação de hidrogênio e sonda $\mathrm{H}^{+}$doadora de ligação de hidrogênio. O tamanho da caixa utilizado foi de 31 ,
15 e $13 \AA$ em relação os eixos X, Y e Z, respectivamente, com diferentes espaçamentos dos pontos e raios de corte. Para reduzir o número de variáveis calculado para cada modelo foram utilizados raios de corte para os campos eletrostático e estereoquímico. Com o objetivo de melhorar a eficiência e diminuir o ruído, foi empregado um filtro para excluir variáveis com variância menor que $2 \mathrm{Kcal} / \mathrm{mol}$. O modelo foi obtido através de análises de PLS utilizando como variáveis dependentes os valores de log $\mathrm{MIC}^{-1}$ frente aos fungos Candida albicans e Cryptococcus neofarmans. $O$ método validação cruzada utilizado foi o LOO.

Nos estudos de QSAR-3D frente à Candida albicans, foram utilizados como grupo de treino os compostos $\mathbf{1}, \mathbf{2}, \mathbf{3}, \mathbf{4}, \mathbf{5}, \mathbf{1 0}, \mathbf{1 1}, \mathbf{1 2} \mathrm{e}$ 13 (Figura 1) e como grupo de teste os compostos 6, 7 e 8 (Figura 1), os quais foram escolhidos de forma aleatória. As melhores condições de análises obtidas encontram-se resumidas na Tabela 1.

Tabela 1. Condições utilizadas na análise CoMFA frente a Candida albicans

\begin{tabular}{ccccccc}
\hline \multirow{2}{*}{ Análise } & \multirow{2}{*}{ Peso } & \multirow{2}{*}{ Sonda } & \multirow{2}{*}{ Carga } & $\begin{array}{c}\text { Intervalo dos } \\
\text { pontos (å) }\end{array}$ & \multicolumn{2}{c}{ Raios de corte $\left(\mathrm{Kcal} / \mathrm{mol}^{-1}\right)$} \\
\hline $\mathbf{1}$ & & & & & estereoquímico eletrostático \\
\hline $\mathbf{2}$ & 0,6 & $\mathrm{O} 2$ & 0 & $1,5: 1,5: 1,5$ & 8 & 16 \\
$\mathbf{3}$ & - & 0 & $1,5: 1,5: 1,5$ & 8 & 16 \\
$\mathbf{4}$ & 0,3 & $\mathrm{O} 3$ & 0 & $1,0: 1,0: 1,0$ & 8 & 16 \\
$\mathbf{6}$ & & $\mathrm{OH} 3$ & +1 & $1,0: 1,0: 1,0$ & 8 & 16 \\
$\mathbf{7}$ & 0,3 & $\mathrm{CH} 3$ & +1 & $1,5: 1,5: 1,5$ & 8 & 16 \\
$\mathbf{8}$ & & $\mathrm{N} 3$ & 0 & $1,0: 1,0: 1,1,0$ & 8 & 16 \\
$\mathbf{9}$ & 0,3 & $\mathrm{~N} 3$ & 0 & $1,0: 1,0: 1,0$ & 8 & 16 \\
\hline
\end{tabular}

Nos estudos de QSAR-3D frente ao Cryptococcus neofarmans fizeram parte do grupo de treino os compostos $2, \mathbf{3}, \mathbf{4}, \mathbf{5}, \mathbf{7}, \mathbf{8}, \mathbf{1 0}$, 11 e 12 (Figura 1) e como grupo de teste os compostos $\mathbf{1}, \mathbf{6}$, e 9 (Figura 1), que foram escolhidos aleatoriamente. As melhores condições de análises obtidas estão resumidamente mostradas na Tabela 2 .

Tabela 2. Condições utilizados na análise CoMFA frente a Cryptococcus neofarmans

\begin{tabular}{ccccccc}
\hline Análise & Peso & \multicolumn{2}{c}{ Sonda Carga $\begin{array}{c}\text { Intervalos dos } \\
\text { pontos (å) }\end{array}$} & $\begin{array}{c}\text { Raios de corte }\left(\mathrm{Kcal}^{2} \mathrm{~mol}^{-1}\right) \\
\text { estereoquímico }\end{array}$ \\
\hline $\mathbf{1}$ & & $\mathrm{CH} 3$ & +1 & $1: 1: 1$ & 8 & 16 \\
$\mathbf{2}$ & 0,3 & $\mathrm{CH} 3$ & +1 & $1: 1: 1$ & 8 & 16 \\
$\mathbf{3}$ & & $\mathrm{CH} 3$ & +1 & $1: 1: 1$ & 0 & 16 \\
$\mathbf{4}$ & 0,3 & $\mathrm{CH} 3$ & +1 & $1: 1: 1$ & 0 & 16 \\
$\mathbf{5}$ & & $\mathrm{O} 2$ & 0 & $1: 1: 1$ & 30 & 30 \\
$\mathbf{6}$ & 0,3 & $\mathrm{O} 2$ & 0 & $1: 1: 1$ & 30 & 30 \\
$\mathbf{7}$ & 0,6 & $\mathrm{O} 2$ & 0 & $1: 1: 1$ & 30 & 30 \\
$\mathbf{8}$ & 0,6 & $\mathrm{O} 2$ & 0 & $1: 1: 1$ & 8 & 16 \\
$\mathbf{1 0}$ & 0,3 & $\mathrm{O} 2$ & 0 & $1: 1: 1$ & 8 & 16 \\
$\mathbf{1 1}$ & 0,3 & $\mathrm{~N} 2$ & 0 & $2: 2: 2$ & 8 & 16 \\
$\mathbf{1 3}$ & & $\mathrm{H}$ & +1 & $1: 1: 1$ & 0 & 16 \\
$\mathbf{1 4}$ & 0,3 & $\mathrm{H}$ & +1 & $1: 1: 1$ & 0 & 16 \\
$\mathbf{1 5}$ & 0,6 & $\mathrm{H}$ & +1 & $1: 1: 1$ & 0 & 16 \\
\hline
\end{tabular}

Os valores de $\log \mathrm{MIC}^{-1}$ frente à Candida albicans foram calculados para os compostos dos grupos de treino, de teste e de validação externa utilizando a análise com maior significância estatística. As atividades biológicas calculadas foram comparadas com os valores descritos na literatura e os mapas de contorno das análises foram construídos. ${ }^{21,41,42,45} \mathrm{O}$ mesmo procedimento foi feito para a análise frente a Cryptococcus neofarmans. 


\section{Validação externa das análises de QSAR-2D e QSAR-3D}

As análises foram validadas utilizando moléculas obtidas da literatura para os modelos de QSAR-2D e QSAR-3D frente a $C$. albicans e C. neofarmans. ${ }^{41,42}$

\section{RESULTADOS E DISCUSSÃO}

\section{Geração da estrutura 3D}

As estruturas geradas no docking foram comparadas com aquelas obtidas por cristalografia de raios X. O cálculo de RMS para as moléculas $\mathbf{3}$ (bis-isopropilfuramidina), 4 (bis-ciclopropilfuramidina), 7 (bis-ciclobutilfuramidina) e $\mathbf{1 1}$ (bis-ciclo-hexilfuramidina) são, respectivamente, 0,$287 ; 0,320 ; 0,447$ e 0,460 . Observando os valores de RMS pode-se concluir que as estruturas dos ligantes obtidas no docking manual estão muito próximas da conformação dos ligantes na estrutura obtida por cristalografia de raios X.

\section{Estudos QSAR-2D frente ao fungo Candida albicans}

A análise de correlação linear expressa numericamente o grau de associação entre as variáveis X e Y. Na análise de RML foi utilizado como variável dependente $\log \mathrm{MIC}^{-1}$ frente a $C$. albicans e como variável independente lipolo Componente Z. Obteve-se a seguinte equação:

$\log \mathrm{MIC}^{-1}=-0,414( \pm 0,25)$ lipolo Componente $\mathrm{Z}+7,26( \pm 0,021)(1)$

$$
\mathrm{R}^{2}=0,69 \quad \mathrm{Q}^{2}=0,51 \quad \mathrm{n}=9 \quad \mathrm{~F}=15,29 \quad \mathrm{~s}=0,257
$$

O parâmetro lipolo é a medida da distribuição lipofílica na molécula e está relacionado com os grupos presentes na molécula em relação aos eixos $\mathrm{X}, \mathrm{Y}$ e Z ${ }^{56} \mathrm{~A}$ Equação 1 indica que a atividade frente a $C$. albicans está correlacionada à distribuição lipofílica da molécula. Quanto menor é o valor desta distribuição maior será a atividade. Os valores de $\mathrm{R}^{2}$ e $\mathrm{Q}^{2}$ foram razoáveis, indicando que o parâmetro lipolo-componente $\mathrm{Z}$ descreve apenas parcialmente a atividade biológica, apesar de ser o parâmetro isolado que descreve melhor a atividade biológica observada. A atividade biológica frente à Candida albicans dos compostos do grupo de treino e do grupo de teste foi calculada utilizando a Equação 1. Os resultados encontram-se na Tabela 3.

O comportamento do composto $\mathbf{8}$ do grupo de teste não foi explicado adequadamente pelo modelo obtido (\% de erro $>$ que $5 \%$ ). As moléculas do grupo de validação externa não tiveram sua atividade explicada de forma adequada (erro $\geq 5 \%$ ). Isto pode ser explicado pelo fato de que essas moléculas são muito diferentes das moléculas do grupo de treino e, assim, seria necessário aumentar o número de moléculas com estruturas diferentes, de modo a ampliar o alcance do modelo obtido.

\section{Estudos QSAR-2D frente ao fungo Cryptococcus neofarmans}

$\mathrm{Na}$ análise de RML utilizando como variável dependente o log $\mathrm{MIC}^{-1}$ frente ao fungo Cryptococcus neofarmans e como variáveis independentes aquelas calculadas previamente com o programa Tsar versão 3.21, obteve-se a Equação 2:

Log $\mathrm{MIC}^{-1}=-0,366( \pm 0,19)$ lipolo componente Z -1,332 $( \pm 0,81)$ índice topológico Balaban $+9,46( \pm 0,90)$

$\mathrm{R}^{2}=0,85 \quad \mathrm{Q}^{2}=0,60 \quad \mathrm{n}=9 \quad \mathrm{~s}=0,195 \quad \mathrm{~F}=17,197$

Os coeficientes e/ou parâmetros da Equação 2 sugerem que quanto menor o parâmetro lipolo-componente $\mathrm{Z}$ e o parâmetro topológico de Balaban maior será a potência do composto frente ao fungo $C$. neofarmans. O índice topológico de Balaban (J) reflete fortemente a ramificação molecular e muito pouco o tamanho molecular, sendo baseado na soma das distâncias de um átomo a outro na conformação da molécula e seu valor é dependente da conformação tridimensional. ${ }^{57,58}$ Assim, segundo a Equação 2, será mais potente a molécula menos ramificada e menor ramificação (menor lipolo-componente Z).

Os valores de $\log \mathrm{MIC}^{-1}$ dos compostos dos grupos de treino, de teste e o de validação lateral foram calculados utilizando a Equação 2. Os resultados encontram-se na Tabela 4.

Tabela 3. Valores reais e calculados de $\log \mathrm{MIC}^{-1}$ frente a Candida albicans dos compostos bisbenzamidínicos dos grupos de treino, de teste e do grupo de validação externa utilizando análise de QSAR-2D

\begin{tabular}{|c|c|c|c|c|c|c|}
\hline Grupo & Molécula & Lipolo-componente Z & $\log \mathrm{MIC}_{\text {real }}^{-1}$ & $\log \mathrm{MIC}^{-1}$ predito & Resíduo & $\%$ erro \\
\hline Treino & 01 & $-0,13$ & 7,68 & 7,31 & 0,37 & 4,82 \\
\hline Treino & 02 & 0,02 & 7,49 & 7,25 & 0,24 & 3,20 \\
\hline Treino & 03 & $-0,01$ & 7,19 & 7,26 & $-0,07$ & 0,97 \\
\hline Treino & 04 & 0,22 & 7,19 & 7,17 & 0,02 & 0,28 \\
\hline Treino & 05 & $-1,72$ & 7,82 & 7,97 & $-0,15$ & 1,92 \\
\hline Treino & 10 & 0,47 & 6,65 & 7,06 & $-0,42$ & 6,32 \\
\hline Treino & 11 & $-0,22$ & 7,57 & 7,35 & 0,22 & 2,91 \\
\hline Treino & 12 & $-1,36$ & 7,73 & 7,82 & $-0,09$ & 1,16 \\
\hline Treino & 13 & 1,02 & 6,73 & 6,84 & $-0,11$ & 1,63 \\
\hline Teste & 06 & 0,36 & 7,22 & 7,11 & 0,11 & 1,51 \\
\hline Teste & 07 & $-0,49$ & 7,82 & 7,46 & 0,36 & 4,57 \\
\hline Teste & 08 & 0,36 & 7,5 & 7,11 & 0,39 & 5,19 \\
\hline Teste & 09 & 1,02 & 6,6 & 6,84 & $-0,24$ & 3,60 \\
\hline validação externa & 14 & 0,37 & 8,05 & 7,11 & 0,94 & 11,72 \\
\hline validação externa & 15 & $-1,57$ & 6,66 & 7,91 & $-1,25$ & 18,77 \\
\hline validação externa & 16 & $-1,18$ & 6,5 & 7,75 & $-1,25$ & 19,21 \\
\hline validação externa & 17 & $-0,07$ & 9,22 & 7,29 & 1,93 & 20,94 \\
\hline validação externa & 18 & 0,53 & 7,47 & 7,04 & 0,43 & 5,75 \\
\hline validação externa & 19 & $-0,48$ & 7,12 & 7,46 & $-0,34$ & 4,76 \\
\hline
\end{tabular}


Tabela 4. Valores reais e calculados de $\log \mathrm{MIC}^{-1}$ frente a Cryptococcus neofarmans dos compostos bisbenzamidínicos dos grupos de treino, de teste e do grupo de validação externa utilizando análise de QSAR-2D

\begin{tabular}{|c|c|c|c|c|c|c|c|}
\hline Grupo & Molécula & $\log \mathrm{MIC}^{-1}{ }_{\text {real }}$ & Lipolo-Z & Balaban & $\log \mathrm{MIC}^{-1}$ predito & Resíduo & \%erro \\
\hline Treino & 03 & 7,79 & $-0,02$ & 1,28 & 7,79 & 0 & 0 \\
\hline Treino & 04 & 8,09 & 0,23 & 0,95 & 8,14 & $-0,04$ & 0,49 \\
\hline Treino & 05 & 8,73 & $-1,72$ & 0,92 & 8,89 & $-0,16$ & 1,83 \\
\hline Treino & 07 & 8,73 & $-0,49$ & 0,94 & 8,41 & 0,32 & 3,67 \\
\hline Treino & 08 & 7,85 & 0,36 & 1,24 & 7,70 & 0,15 & 1,91 \\
\hline Treino & 10 & 7,55 & 0,47 & 1,28 & 7,61 & $-0,06$ & 0,79 \\
\hline Treino & 11 & 8,48 & $-0,23$ & 0,91 & 8,34 & 0,12 & 1,42 \\
\hline Treino & 12 & 8,03 & $-1,36$ & 1,40 & 8,12 & 0,09 & 1,12 \\
\hline Treino & 13 & 7,74 & 1,02 & 0,86 & 7,97 & $-0,23$ & 2,97 \\
\hline Teste & 01 & $-0,130$ & 1,289 & 7,39 & 7,72 & $-0,33$ & 4,49 \\
\hline Teste & 02 & 0,025 & 1,258 & 8,4 & 7,82 & 0,58 & 6,91 \\
\hline Teste & 06 & 0,234 & 0,946 & 8,75 & 8,31 & 0,44 & 5,01 \\
\hline Teste & 09 & 0,725 & 0,968 & 8,13 & 8,46 & $-0,33$ & 4,08 \\
\hline validação externa & 14 & 0,375 & 1,124 & 8,05 & 8,13 & $-0,08$ & 0,95 \\
\hline validação externa & 15 & $-1,567$ & 1,113 & 7,56 & 7,43 & 0,13 & 1,72 \\
\hline validação externa & 16 & $-1,181$ & 1,389 & 7,40 & 7,20 & 0,20 & 2,65 \\
\hline validação externa & 17 & $-0,065$ & 1,289 & 8,30 & 7,75 & 0,55 & 6,68 \\
\hline validação externa & 18 & 0,527 & 1,492 & 7,13 & 7,69 & $-0,56$ & 7,88 \\
\hline validação externa & 19 & $-0,480$ & 1,363 & 8,02 & 7,49 & 0,53 & 6,55 \\
\hline
\end{tabular}

Tabela 5. Resultados estatísticos da análise CoMFA frente ao fungo Candida albicans

\begin{tabular}{|c|c|c|c|c|c|c|c|}
\hline \multirow{2}{*}{ Análise } & \multirow{2}{*}{$\begin{array}{l}\text { Número ótimo de } \\
\text { componentes }\end{array}$} & \multirow{2}{*}{$\mathrm{R}^{2}$} & \multirow{2}{*}{$\mathrm{Q}^{2}$} & \multirow{2}{*}{$\mathrm{s}$} & \multirow{2}{*}{$\mathrm{F}$} & \multicolumn{2}{|c|}{ Contribuição } \\
\hline & & & & & & Estéreoquímico & eletrostático \\
\hline 1 & 1 & 0,790 & 0,280 & 0,210 & 26,40 & 0,941 & 0,059 \\
\hline 2 & 1 & 0,836 & 0,704 & 0,185 & 35,69 & 0,948 & 0,052 \\
\hline 3 & 1 & 0,802 & 0,225 & 0,203 & 28,43 & 0,936 & 0,064 \\
\hline 4 & 1 & 0,820 & 0,600 & 0,194 & 31,80 & 0,955 & 0,045 \\
\hline 5 & 1 & 0,824 & 0,281 & 0,190 & 32,82 & 0,966 & 0,034 \\
\hline 6 & 1 & 0,824 & 0,603 & 0,192 & 32,82 & 0,966 & 0,034 \\
\hline 7 & 1 & 0,801 & 0,540 & 0,204 & 28,09 & 0,955 & 0,045 \\
\hline 8 & 1 & 0,800 & 0,240 & 0,205 & 27,91 & 0,942 & 0,058 \\
\hline 9 & 1 & 0,827 & 0,608 & 0,190 & 33,55 & 0,95 & 0,045 \\
\hline
\end{tabular}

\section{Estudos de QSAR-3D frente ao fungo Candida albicans}

Os resultados da análise por metodologia CoMFA encontram-se na Tabela 5.

As condições utilizadas na análise 2 (Tabela 2) foram as que levaram aos melhores resultados estatísticos. Assim, foram calculados os valores de log $\mathrm{MIC}^{-1}$ para os compostos dos grupos de treino, de teste e do grupo de moléculas utilizadas na validação externa. Os resultados obtidos estão descritos na Tabela 6 .

Adicionalmente, os mapas de contorno utilizando-se as condições da citada análise 2 (Tabela 2) foram construídos (Figura 2). As áreas vermelhas são regiões onde grupos com um potencial eletrostático negativo levam ao aumento da potência e as áreas azuis são regiões onde grupos com potencial eletrostático positivo levam a um aumento da potência. As áreas verdes são regiões onde grupos volumosos são favoráveis (maior potência) e as áreas amarelas onde grupos volumosos são indesejáveis, pois diminuem a potência.

A análise de QSAR-3D indica que o fator estereoquímico é um fator importante para a atividade biológica frente ao fungo Candida albicans. A presença de grupos muito volumosos no grupo amidínico diminui a potência. O grupo carregado positivamente também é importante para a atividade biológica, o que está de acordo com as
Tabela 6. Valores calculados de log $\mathrm{MIC}^{-1}$ para os compostos do grupo de treino frente a Candida albicans utilizando as condições da análise 2- CoMFA

\begin{tabular}{lccccc}
\hline Grupo & Molécula & $\log \mathrm{MIC}^{-1}{ }_{\text {real }} \log \mathrm{MIC}^{-1}{ }_{\text {predito }}$ & Resíduo & \%erro \\
\hline treino & 01 & 7,68 & 7,64 & $-0,04$ & 0,52 \\
treino & 02 & 7,49 & 7,29 & $-0,2$ & 2,67 \\
treino & 03 & 7,19 & 7,23 & 0,04 & 0,56 \\
treino & 04 & 7,19 & 7,46 & 0,27 & 3,76 \\
treino & 05 & 7,82 & 7,65 & $-0,17$ & 2,17 \\
treino & 10 & 6,65 & 6,72 & 0,07 & 1,05 \\
treino & 11 & 7,57 & 7,31 & $-0,26$ & 3,43 \\
treino & 12 & 7,73 & 7,92 & 0,19 & 2,46 \\
treino & 13 & 6,73 & 6,83 & 0,1 & 1,49 \\
teste & 06 & 7,22 & 7,17 & $-0,35$ & 4,65 \\
teste & 07 & 7,82 & 7,41 & $-0,41$ & 5,24 \\
teste & 08 & 7,5 & 7,36 & $-0,14$ & 1,87 \\
validação externa & 14 & 8,05 & 7,42 & $-0,63$ & 7,83 \\
validação externa & 15 & 6,66 & 7,41 & 0,75 & 11,26 \\
validação externa & 16 & 6,5 & 7,87 & 1,38 & 21,26 \\
validação externa & 17 & 9,22 & 7,55 & $-1,66$ & 18,02 \\
validação externa & 18 & 7,47 & 7,66 & 0,22 & 2,96 \\
validação externa & 19 & 7,12 & 7,49 & 0,37 & 5,20 \\
\hline
\end{tabular}


Tabela 7. Resultados da análise CoMFA frente a Cryptococcus neofarmans

\begin{tabular}{|c|c|c|c|c|c|c|c|c|}
\hline \multirow{2}{*}{ Análise } & \multirow{2}{*}{$\mathrm{R}^{2}$} & \multirow{2}{*}{$\mathrm{Q}^{2}$} & \multirow{2}{*}{$\begin{array}{l}\mathrm{N}^{\circ} \text { ótimo de } \\
\text { componentes }\end{array}$} & \multirow{2}{*}{$\mathrm{N}$} & \multirow{2}{*}{$\mathrm{F}$} & \multirow{2}{*}{ S } & \multicolumn{2}{|c|}{ contribuição } \\
\hline & & & & & & & Estéreoquímico & Eletrostático \\
\hline Análise 3 & 0,987 & 0,582 & 2 & 10 & 264,9 & 0,058 & 0,950 & 0,050 \\
\hline Análise 7 & 0,990 & 0,710 & 2 & 10 & 336,7 & 0,052 & 0,950 & 0,050 \\
\hline Análise 8 & 0,998 & 0,808 & 2 & 10 & 278,3 & 0,057 & 0,979 & 0,021 \\
\hline Análise 4 & 0,958 & 0,623 & 1 & 10 & 79,09 & 0,105 & 0,948 & 0,052 \\
\hline Análise 14 & 0,794 & 0,474 & 1 & 10 & 30,899 & 0,216 & 0 & 1 \\
\hline Análise 15 & 0,934 & 0,523 & 2 & 10 & 49,560 & 0,131 & 0 & 1 \\
\hline
\end{tabular}
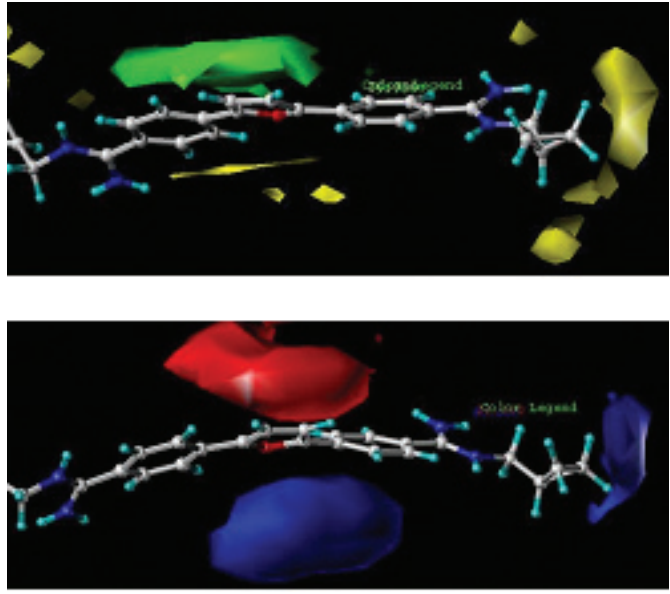

Figura 2. Mapas de contorno frente ao fungo Candida albicans. Sonda $\mathrm{O} 2$ carga $\mathrm{O}$

informações da literatura. ${ }^{25,26}$ Como observado na análise de QSAR$2 \mathrm{D}$, as moléculas do grupo de validação externa não foram bem explicadas pelo modelo (erro $>5 \%$ ). Isto pode ser explicado pela pequena variação estrutural do grupo de treino.

\section{Estudos QSAR-3D frente ao fungo Cryptococcus neofarmans}

Os resultados da análise do CoMFA frente a Cryptococcus neofarmans encontram-se na Tabela 7.

Utilizando as condições da análise 8 (Tabela 3), obtiveram-se os melhores resultados estatísticos e foram calculados os valores de $\log \mathrm{MIC}^{-1}$ para os compostos do grupo de treino, para o grupo de teste e para o grupo de moléculas utilizadas na validação externa. Os resultados encontram-se na Tabela 8.

Os mapas de contorno foram construídos (Figura 3) também se utilizando as condições descritas na análise 8 (Tabela 3). Nas áreas vermelhas mostram-se regiões onde um potencial eletrostático negativo é favorável para a atividade e nas áreas azuis são desfavoráveis para a atividade (ou seja, menor potência). As áreas verdes são regiões onde grupos volumosos são favoráveis (maior potência) e as áreas amarelas onde grupos volumosos são indesejáveis, pois diminuem a potência.

A análise do CoMFA está de acordo com a análise de QSAR-2D, uma vez que nas duas análises o parâmetro estereoquímico foi importante para a descrição da atividade biológica. Na análise do CoMFA a presença de grupos volumosos no grupo amidínico é desfavorável para a atividade biológica.

Como todas as moléculas do banco de dados são carregadas positivamente, o mapa eletrostático gerado não foi muito útil para a predição da atividade biológica. No entanto, o potencial positivo no anel furânico pode indicar a possibilidade de ligação de hidrogênio com os grupos fosfatos, carregados negativamente, presentes na parte externa da fenda do B-DNA. ${ }^{59}$
Tabela 8. Valores calculados de $\log \mathrm{MIC}^{-1}$ para os compostos do grupo de treino, do grupo de teste e validação externa frente a Cryptococcus neofarmans utilizando as condições da análise 8- CoMFA

\begin{tabular}{lccccc}
\hline Grupo & Molécula & $\log$ MIC $^{-1}{ }_{\text {real }}$ & $\log$ MIC $^{-1}{ }_{\text {predito }}$ & Resíduo & \%erro \\
\hline treino & 02 & 8,4 & 8,37 & 0,03 & 0,30 \\
treino & 03 & 7,79 & 7,87 & 0,08 & 1,01 \\
treino & 04 & 8,81 & 8,88 & 0,07 & 0,83 \\
treino & 05 & 8,73 & 8,62 & 0,11 & 1,23 \\
treino & 07 & 8,73 & 8,72 & 0,01 & 0,10 \\
treino & 08 & 7,85 & 7,84 & 0,01 & 0,17 \\
treino & 10 & 7,55 & 7,60 & 0,05 & 0,61 \\
treino & 11 & 8,48 & 8,46 & 0,02 & 0,25 \\
treino & 12 & 8,03 & 8,18 & 0,15 & 1,85 \\
Teste & 01 & 7,39 & 7,92 & 0,53 & 6,67 \\
Teste & 06 & 8,13 & 7,56 & 0,57 & 7,61 \\
Teste & 09 & 7,74 & 8,24 & 0,50 & 6,04 \\
validação externa & 14 & 8,05 & 7,61 & 0,44 & 5,75 \\
validação externa & 15 & 7,56 & 8,18 & 0,62 & 7,62 \\
validação externa & 16 & 7,4 & 8,18 & 0,78 & 9,56 \\
validação externa & 17 & 8,3 & 7,77 & 0,53 & 6,79 \\
validação externa & 18 & 7,13 & 8,07 & 0,94 & 11,64 \\
validação externa & 19 & 8,02 & 8,07 & 0,05 & 0,60 \\
\hline
\end{tabular}
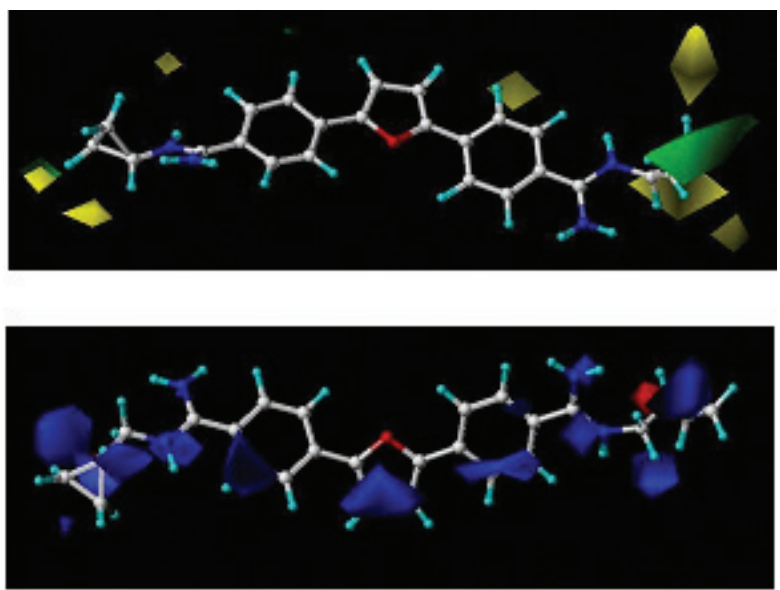

Figura 3. Mapas de contorno frente ao fungo Cryptococcus neofarmans Sonda $\mathrm{CH}_{3}^{+1}$

\section{CONCLUSÕES}

Um dos problemas da análise de QSAR é a escolha da conformação a ser utilizada. Normalmente, a conformação do ligante escolhida é aquela observada na estrutura de raios $\mathrm{X}$ no complexo ligante-alvo biológico, no entanto, muitas vezes não temos esta estrutura de todos os ligantes no complexo. O docking manual mostrou ser uma técnica 
adequada para se obter a conformação semelhante àquela observada pela cristalografia de raios $\mathrm{X}$, uma vez que as estruturas dos ligantes testados obtidas no docking manual estão muito próximas da conformação dos ligantes na estrutura de raios X.

As análises de QSAR-2D e QSAR-3D permitiram inferir que os parâmetros estereoquímicos e lipofílicos são importantes para a atividade biológica frente à Candida albicans e Cryptococcus neofarmans.

As análises de QSAR-2D indicaram que a atividade frente à Candida albicans está correlacionada à distribuição lipofílica da molécula. No entanto, este parâmetro isolado não explica de forma adequada a atividade biológica observada. O parâmetro lipofílico está relacionado com as características farmacodinâmicas necessárias à absorção. A equação obtida da análise de QSAR-2D frente ao fungo Cyptococcus neofarmans indica que a distribuição lipofílica na molécula e a ramificação presente são importantes para a atividade biológica. Quanto menor o valor do lipolo-componente $\mathrm{Z}$ e quanto menos ramificada for a molécula e menor for esta ramificação, mais potente será a molécula.

As análises de QSAR-3D mostraram que o fator estereoquímico também é um fator importante para a atividade frente aos dois fungos. A presença de grupos muito volumosos no grupo amidínico diminui a potência dos compostos bis-benzamidínicos. Isto já era esperado, uma vez que estudos prévios de modelagem molecular e relação estrutura-atividade utilizando compostos amidínicos indicam a importância da estrutura isoélica à fenda menor do B-DNA destes compostos para a interação.

\section{AGRADECIMENTOS}

À FAPEMIG (CEX PPM III 0207/09, PRONEX EDT 479/07), CAPES e ao CNPq pelo auxílio financeiro.

\section{REFERÊNCIAS}

1. Tortora, G. J.; Funke, B. R.; Case, C. L.; Microbiologia, $6^{\mathrm{a}}$ ed., ArtMed: Porto Alegre, 2000.

2. Lacaz, C. S.; Porto, E.; Martins, J. E. C.; Heins-Vaccari, E. M.; Melo, N. T.; Tratado de Micologia Médica Lacaz, $9^{\text {a }}$ ed., Sarvier: São Paulo, 2001.

3. Harbarth, S.; Samore, M. H.; Emerg. Infect. Dis. 2005, 11, 794.

4. McGowan, J. E.; Tenover, F. C.; Nat. Rev. Microbiol. 2004, 2, 251.

5. Anaissie, E.; Clin. Infect. Dis. 1992, 14, S43.

6. Hadley, S.; Karchmer, A. W.; Infect. Dis. Clin. N. Am. 1995, 9, 1045.

7. Sundriyal, S.; Sharma, R. K.; Jain, R.; Curr. Med. Chem. 2006, 13, 1321.

8. Perfect, J. R.; Marr, K. A.; Walsh, T. J.; Greenberg, R. N.; DuPont, B.; De la Torre-Cisneros, J.; Just-Nubling, G.; Schlamm, H. T.; Lutsar, I.; Espinel-Ingroff, A.; Johnson, E.; Clin. Infect. Dis. 2003, 36, 1122.

9. Espinel-Ingroff, A.; J. Clin. Microbiol. 2001, 39, 954.

10. Pfaller, M. A.; Diekema, D. J.; J. Clin Microbiol. 2004, 42, 4419.

11. Walsh, T. J.; Petraitis, V.; Petraitiene, R.; Field-Ridley, A.; Sutton, D.; Mahmoud, G.; Tin, S.; Schaufele, R.; Peter, J.; Bacher, J.; Casler, H.; Armstrong, D.; Espinel-Ingroff, A.; Rinaldi, M. G.; Lyman, C. A. ; J. Infec. Dis. 2003, 188, 305.

12. Gurgui, M.; Munoz, P.; Enf. Infec. Microbiol. Clin. 2007, 25, 587.

13. Del Palacio, A.; Alhambra, A.; Cuetara, M. S.; Ponton J.; Rev. Iberoam. Micol. 2007, 24, 187.

14. Marques, M. B.; Cadernos de Saúde Pública 1995, 11, 361.

15. Colombo, A. L.; Guimarães, T.; Rev. Soc. Bras. Med. Trop. 2003, 36, 599.

16. Scorzoni, L.; Benaducci, T.; Almeida, A. M. F.; Silva, D. H. S.; Bolzani, V. S.; Gianinni, M. J. S.; Braz. J. Microbiol. 2007, 38, 391.

17. Gonzalez, C. E.; Venzon, D.; Lee, S.; Mueller, B. U.; Pizzo, P. A.; Walsh, T.; J. Clin. Infect. Dis. 1996, 23, 515.
18. Wilson, W. D.; Tanious, F. A.; Mathis, A.; Tevis, D.; Hall, J. E.; Boykin, D. W.; Biochimie 2008, 90, 999.

19. Athri, P.; Wenzler, T.; Ruiz, P.; Brun, R.; Boykin, D. W.; Tidwell, R.; Wilson, W. D.; Bioorg. Med. Chem. 2006, 14, 3144.

20. Kuryshev, Y. A.; Ficker, E., Wang, L.; Hawryluk, P.; Dennis, A. T.; Wible, B. A.; Brown, A. M.; Kang, J.; Chen, X. L.; Sawamura, K.; Reynolds W.; Rampe, D.; J. Pharmacol. Exp. Ther. 2005, 312, 316.

21. Tidwell, R. R.; Jones, S. K.; Naiman, N. A.; Berger, L. C.; Brake, W. B.; Dystra, C. C.; Hall, J. E.; Antimicrob. Agents Chemother. 1993, 37, 1713.

22. Lombardy, R. L.; Tanious, F. A.; Ramachandrew, R.; Tidwell, R. R.; Wilson, W. W.; J. Med. Chem. 1996, 34, 1452.

23. Edwards, K. J.; Jenkins, T. C.; Neidle, S.; Biochemistry 1992, 31, 7104.

24. Hall, J. E.; Kerrigan, J. E.; Ramachandran, K.; Bender, B. C.; Stanko, J. P.; Jones, S. K.; Patrick, D. A.; Tidwell, R. R.; Antimicrob. Agents Chemother. 1998, 42, 666.

25. Boykin, D. W.; J. Braz. Chem. Soc. 2002, 13, 763.

26. Ngujen, B.; Hamelberg, D.; Bailly, C.; Colson, P.; Stanek, J.; Brun, R.; Biophys. J. 2004, 88, 1028.

27. Neidle, S.; Nat. Prod. Rep. 2001, 18, 291.

28. Montanari, C. A.; Trent, J. O.; Jenkins, T. C.; J. Braz. Chem. Soc. 1998, 9, 175.

29. Oliveira, A. M.; Custódio, F. B.; Donnici, C. L.; Montanari, C. A.; Eur. J. Med. Chem. 2003, 38, 141.

30. Campbell, N. H.; Evans, D.; Lee, M. P. H.; Parkinson, G. N.; Neidle, S.; Bioorg. Med. Chem. Lett. 2006, 16, 5.

31. Montanari, C. A.; Tute, M. S.; Beezer, A. E.; Mitchell, J. C.; J. Comput. Aided Mol. Des. 1996, 10, 67.

32. Keseru, G.; Makaro, G. M.; Drug Discovery Today 2006, 11, 741.

33. Ferreira, M. M. C.; Montanari, C. A.; Gáudio, A. C.; Quim. Nova 2002, $25,439$.

34. Correia, P. R. M.; Ferreira, M. M. C.; Quim. Nova 2007, 30, 481.

35. Gáudio, A.; Zandonade, E.; Quim. Nova 2001, 24, 658.

36. Del Poeta, M.; Schell, W. A.; Dykstra, C. C.; Jones, S. K.; Tidwell, R. R.; Kumar, A.; Boykin, D. W.; Perfect, J. R.; Antimicrob. Agents Chemother. 1998, 42, 2503.

37. Del Poeta, M; Schell, W. A.; Dykstra C. C.; Jones, S. K., Tidwell, R. R.; Czarny, A.; Bajic, M.; Kumar, A.; Boykin, D. W.; Perfect, J. R.; Antimicrob. Agents Chemother. 1998, 42, 2495.

38. SYBYL 6.7 ed, SYBYL molecular modeling software; Tripos Associates Ltd., St Louis, 2000.

39. Muegge, I.; Oloff, S.; Drug discovery today: technologies 2006, 3, 405.

40. Cramer, R. D.; Patterson, D. E.; Bunce, J. D.; J. Am. Chem. Soc. 1988, $110,5959$.

41. Golbraikh, A.; Bernard, P.; Chretien, J. R.; Eur. J. Med. Chem. 2000, 35, 123.

42. Jayatilleke, P. P. N.; Nair, A. C.; Zauhar, R.; Welsh, W. J.; J. Med. Chem. 2000, 43, 4446.

43. Boykin, D. W.; Kumar, A.; Xiao, G. E.; Wilson, W. D.; Bender, B. C.; McCurdy, D. R.; Hall, J. E.; Tidwell, R. R.; J. Med. Chem. 1998, 41, 124.

44. Dykstra, C. C.; McClernon, D. R.; Elwell, L. P.; Tidwell, R. R.; Antimicrob. Agents Chemother. 1994, 38, 1890.

45. Hopkins, K. T.; Wilson, W. D.; Bender, B. C.; McMudy, D. R.; Hall, J. E.; Tidwell, R. R.; Kumar, A.; Bajic, M.; Boykin, D. W.; J. Med. Chem. 1998, 41, 3872.

46. MOPAC v. 7.0; University of Indiana, Bloomington, USA, IN 47405.

47. Rocha, R. K.; Dissertação de Mestrado, Universidade Federal de Minas Gerais, Brasil, 1998.

48. MacroModel v. 5.5; Departament of Chemistry, Columbia University, New York, USA, 10027.

49. Weiner, S. J.; Kolman, P. A.; Care, D. A.; Singh, V. C.; Ghio, C.; Alagon, G.; Profeta, S.; Weiner, P.; J. Am. Chem. Soc. 1984, 106, 765. 
50. Dewar, M. J. S.; Thiel, W.; J. Am. Chem. Soc. 1977, 99, 4899.

51. Evans, D.; Neidle, S.; J. Med. Chem. 2006, 49, 4232.

52. http://www.rcsb.org/pdb/home/home.do, acessada em Maio 2005 e Junho 2010.

53. Biosym Technologies. Insight II Version 2.3.0 User Guide, Part 1; Biosym, San Diego, USA, 1995.

54. Tsar 3.21, Reference Guide, Oxford Molecular Limited, Accelyrs, 2000.
55. Kubinyi, H.; The Regression Analysis: Program BILIN, disponível em http://www.kubinyi.de/bilin-program.html, acessada em Junho 2009.

56. Pedretti, A.; Villa L.; Vistoli, G.; J. Med. Chem. 2002, 45, 1460.

57. Montanari, C. A.; Cass, Q. B.; Tiritan, M. E.; Souza, A. L. S. S.; Anal. Chim. Acta 2000, 419, 93.

58. Balaban, A. T.; Chem. Phys. Lett. 1982, 89, 399.

59. Wang, H.; Laughton, C. A.; Methods 2007, 42, 196. 